\title{
Information Loss in Black Hole Evaporation
}

\author{
Jingyi Zhang, ${ }^{*}$ Yapeng Hu, Zheng Zhao ${ }^{\dagger}$ \\ Department of Physics , Beijing Normal University, Beijing 100875, China
}

(Dated: July 25, 2018)

\begin{abstract}
Parikh-Wilczek tunnelling framework is investigated again. We argue that Parikh-Wilczek's treatment, which satisfies the first law of black hole thermodynamics and consists with an underlying unitary theory, is only suitable for a reversible process. Because of the negative heat capacity, an evaporating black hole is a highly unstable system. That is, the factual emission process is irreversible, the unitary theory will not be satisfied and the information loss is possible.
\end{abstract}

PACS number(s): 04.70.Dy

Keywords: generalized second law of thermodynamics, Hawking radiation, information puzzle

In 1975, Stephen Hawking Published his astounding discovery that black holes radiate thermally. With the emission of Hawking radiation, the black hole could lose energy, shrink, and eventually evaporate completely. Nevertheless, it sets up a disturbing and difficult problem: what happens to information during the black hole evaporation? About this puzzle, there are two opposite opinions. Hawking and Kip Thorne thought that information is lost in black hole evaporation. But John Preskill believes that information is not lost and can get out of a black hole. In 2004, Hawking changed his opinion. He thinks that information can get out of a black hole[1]. In 2000, Parikh and Wilczek investigated the black hole radiation again. They treat Hawking radiation as a tunnelling process, and give a semiclassical but the first explicit calculation about this problem [2]. Their result is considered to be in agreement with an underlying unitary theory and a support to the information conservation. Following this method, a number of static or stationary rotating black holes are studied [3, 4, 5, 6, 7, 8, 9, 10, 11, 12, 13, 14, 15, 16, 17, 18]. The same result, that is, Hawking radiation is no longer pure thermal, unitary theory is satisfied and information is conserved, is obtained. Is information conserved during the process of black hole radiation? Does the Parikh-Wilczek tunnelling framework prove the information conservation? In this letter, we argue that Parikh-Wilczek tunnelling framework is only suitable for the reversible process. Considering the factual emission process is an irreversible process, information loss is possible.

We first investigate the tunnelling process from the Schwarzschild black hole. According to Ref. 2], the imaginary part of the action for the classically forbidden trajectory is

$$
\operatorname{Im} S=\operatorname{Im} \int_{r_{i}}^{r_{f}} p_{r} \mathrm{~d} r=\int_{M}^{M-\omega}-2 \pi r_{H}^{\prime} \mathrm{d} M^{\prime}=-\frac{1}{2}\left[4 \pi(M-\omega)^{2}-4 \pi M^{2}\right]=-\frac{1}{2}\left(S_{f}-S_{i}\right),
$$

\footnotetext{
* E-mail: physicz@tom.com

$\dagger$ E-mail: zhaoz43@hotmail.com
} 
and the emission rate is therefore 2,19$]$

$$
\Gamma \sim e^{-2 \operatorname{Im} S}=e^{\triangle S_{B H}}
$$

which is in agreement with an unitary result in Quantum Mechanics, $\Gamma(i \rightarrow f)=\left|M_{f i}\right|^{2} \cdot e^{\triangle S}$. Therefore, ParikhWilczek tunnelling framework is considered to be a support to the information conservation. We rewrite equation (1) as

$$
\operatorname{Im} S=\operatorname{Im} \int_{r_{i}}^{r_{f}} p_{r} \mathrm{~d} r=\int_{M}^{M-\omega}-\frac{1}{2} \cdot \frac{\mathrm{d} M^{\prime}}{T^{\prime}}=-\int_{S_{i}}^{S_{f}} \frac{1}{2} \mathrm{~d} S^{\prime}=-\frac{1}{2}\left(S_{f}-S_{i}\right),
$$

where $T^{\prime}=\frac{1}{8 \pi M^{\prime}}$ is the Hawking temperature, $S_{i}$ and $S_{f}$ are the Bekenstein-Hawking entropy of the black hole corresponding to the initial state and the final state, respectively. From equation (3) we see that the emission process satisfies the first law of black hole thermodynamics, that is

$$
\frac{\mathrm{d} M^{\prime}}{T^{\prime}}=\mathrm{dS}^{\prime}
$$

In fact, similar relationship can also be found in the stationary rotating black holes. Let us, for example, take into account the tunnelling of an uncharged particle from the Kerr-Newman black hole. The imaginary part of the action for the classically forbidden trajectory is [16]

$$
\begin{aligned}
\operatorname{Im} S & =\operatorname{Im} \int_{t_{i}}^{t_{f}}\left(L-p_{\varphi} \dot{\varphi}\right) \mathrm{d} t \\
& =-\frac{1}{2} \int_{M}^{M-\omega}\left[\frac{4 \pi\left(M^{\prime 2}+M^{\prime} \sqrt{M^{\prime 2}-a^{2}-q^{2}}-\frac{1}{2} q^{2}\right)}{\sqrt{M^{\prime 2}-a^{2}-q^{2}}} \mathrm{~d} M^{\prime}-\frac{4 \pi\left(M^{\prime 2}+M^{\prime} \sqrt{M^{\prime 2}-a^{2}-q^{2}}-\frac{1}{2} q^{2}\right)}{\sqrt{M^{\prime 2}-a^{2}-q^{2}}} \Omega_{H}^{\prime} \mathrm{d} J^{\prime}\right] \\
& =-\frac{\pi}{2}\left[\left((M-\omega)+(M-\omega) \sqrt{(M-\omega)^{\prime 2}-a^{2}-q^{2}}\right)^{2}-\left(M+M \sqrt{M^{\prime 2}-a^{2}-q^{2}}\right)^{2}\right] \\
& =-\frac{1}{2}\left(S_{f}-S_{i}\right) .
\end{aligned}
$$

Similarly, we rewrite equation (5) as the following

$$
\operatorname{Im} S=-\frac{1}{2} \int_{M}^{M-\omega}\left[\frac{\mathrm{d} M^{\prime}}{T^{\prime}}-\frac{\Omega_{H}^{\prime} \mathrm{d} J^{\prime}}{T^{\prime}}\right]=-\int_{S_{i}}^{S_{f}} \frac{1}{2} \mathrm{dS}^{\prime}=-\frac{1}{2}\left(S_{f}-S_{i}\right),
$$

where

$$
T^{\prime}=\frac{\sqrt{M^{\prime 2}-a^{2}-q^{2}}}{4 \pi\left(M^{\prime 2}+M^{\prime} \sqrt{M^{\prime 2}-a^{2}-q^{2}}-\frac{1}{2} q^{2}\right)} .
$$

Obviously, from equation (6) we can easily find the relationship

$$
\frac{\mathrm{d} M^{\prime}}{T^{\prime}}-\frac{\Omega_{H}^{\prime} \mathrm{d} J^{\prime}}{T^{\prime}}=\mathrm{d} \mathrm{S}^{\prime}
$$

In fact, Eq. (4) and Eq. (8) are the differential forms of the first law of black hole thermodynamics [20]. It is an incorporation of the energy conservation law, $\mathrm{d} M-\Omega_{H} \mathrm{~d} J=\mathrm{d} Q$, and the second law of thermodynamics, $\mathrm{d} S=\frac{\mathrm{d} Q}{T}$. For a Schwarzschild black hole, there is no angular momentum, the first law of the black hole thermodynamics is $d M=d Q$. The equation of energy conservation is suitable for any process (reversible or irreversible process). But the equation $\mathrm{d} S=\frac{\mathrm{d} Q}{T}$ is only true for a reversible process. For an irreversible process, $\mathrm{d} S>\frac{\mathrm{d} Q}{T}$. That is, Parikh-Wilczek 
tunnelling framework has treated the emission process as an reversible process. In their treatment, the black hole and the outside approach an thermal equilibrium. There is an entropy flux $\mathrm{d} S=\frac{\mathrm{d} Q}{T}$ between the black hole and the outside. As the black hole radiate, the entropy of the black hole decreases, but the total entropy of the black hole and the outside is constant, and therefore the information is conserved. Thus, in Parikh-Wilczek tunnelling framework the result is consistent with an unitary theory. But, in fact, because of the negative heat capacity, an evaporating black hole (a Schwarzschild black hole or a Kerr-Newman black hole) is (when is isolation) a highly unstable system. With the radiation, the thermal equilibrium between the black hole and the outside is unstable. There will be a difference in temperature, that is, the process is irreversible. In this case, we can not get the relationship $\mathrm{d} S=\frac{\mathrm{d} Q}{T}$. Then the equations (11) and (5) will not be satisfied. That is, the emission rate from the Schwarzschild black hole or from the Kerr-Newman black hole will not be in agreement with the unitary theory. The total entropy will increase, and therefore information is lost during the process of the black hole radiation. That is to say, Parikh-Wilczek tunnelling framework has not proved the information conservation.

In fact, there are two clues for us to comprehend the information paradox. One clue is that in physics there are many conservation laws, such as the energy conservation law, the momentum conservation law and the electric charge conservation law, but there is no information conservation law. On the contrary, if the viewpoint in Information Theory, which treats information as negative entropy, is correct, and if the information entropy and the thermodynamical entropy are the same in essence, then, the information loss is a natural result. This is because of the second law of thermodynamics-the principle of entropy increase for an irreversible process. Now that the entropy is not conserved, of course, information is not conserved either.

Another clue is that the treatments before in black hole radiation, as pointed out by Hawking in 2004 [21], are too idealization. Hawking radiation, in practice, may deviate from the perfect thermal spectrum, and maybe some "slag" is left in the final state of the black hole evaporation. In conclusion, a factual Hawking process does not conserved in information. But part of the information can get out of the black hole or stay at the final state of the black hole.

\section{Acknowledgments}

This research is supported by National Natural Science Foundation of China (Grant No. 10373003) and National Basic Research Program of China (Grant No. 2003CB716300).

[1] S. W. Hawking, arXiv: hep-th/0507171

[2] M. K. Parikh, F. Wilczek, Phys. Rev. Lett., 85, 5042(2000) [arxiv: hep-th/9907001.

[3] M. K. Parikh, Int. J. Mod. Phys. D 13,2355(2004) [arXiv: hep-th/0405160.

[4] S. Hemming, E. Keski-Vakkuri, Phys. Rev.D64, 044006(2001).

[5] A. J. M. Medved, Phys. Rev.D66, 124009(2002).

[6] M. A.ves, Int. J. Mod. Phys. D 10, 575(2001).

[7] E. C. Vagenas, Phys. Lett. B 503,399(2001). 
[8] E. C. Vagenas, Phys. Lett. B 533,302(2002).

[9] E. C. Vagenas, Mod. Phys. Lett. A17,609(2002).

[10] E. C. Vagenas, Phys. Lett. B 559,65(2003).

[11] E. C. Vagenas, Phys. Lett. B 584,127(2004).

[12] E. C. Vagenas, Mod. Phys. Lett. A20,2449(2005).

[13] M. Arzano, A.J.M. Medved, E. C. Vagenas, J. High Energy Phys., JHEP09(2005)037.

[14] M. R. Setare, E. C. Vagenas, arXiv: hep-th/0405186

[15] J. Zhang, Z. Zhao, J. High Energy Phys., JHEP10(2005)055

[16] J. Zhang, Z. Zhao, Phys. Lett. B 618, 14(2005).

[17] J. Zhang, Z. Zhao, Mod. Phys. Lett. A 20,1673(2005).

[18] J. Zhang, Z. Zhao, Nucl. Phys. B 725,173(2005).

[19] E. Keski-Vakkuri, P. Kraus, Nucl. Phys. B 491, 249 (1997) arXiv:hep-th/9610045.

[20] J. M. Bardeen, B. Carter, S. W. Hawking, Commun. Math. Phys. 31, 161(1973).

[21] S. W. Hawking, Speech at 17th international conference on GRG. Dublin(21 $1^{\text {st }}$, July, 2004). 